\title{
High Risk Pregnancy
}

\author{
Surya Malik*1 and Sushma Sinha ${ }^{2}$ \\ ${ }^{1}$ Specialist, Obstetrics \& Gynecology, King Khalid Hospital, Saudi Arabia \\ ${ }^{2}$ Senior Specialist \& Head of Department, India
}

Received: October05, 2017; Published: October 13, 2017

*Corresponding author : Surya Malik, Specialist, Obstetrics \& Gynecology, King Khalid Hospital, Alkharj, Riyadh, Saudi Arabia

Abstract

\section{"Let every mother and child to be counted"}

Motherhood is a natural phenomenon, but safe motherhood is fraught with many complications related to of pregnancy and child birth. A "high risk pregnancy"(HRP) is one in which the maternal environment or past reproductive performance presents a significant risk to fetal wellbeing, such as premature birth, small for date infant, full term with low reservoir or still births and early neonatal deaths. Identification of patients at risk for these complicated pregnancies with poor outcome is fundamental to antenatal care. A woman is considered to have a high risk pregnancy when health concerns exist that may threaten the natural course of the development or birth of the baby, or that pose a risk to the mother. In such cases the mother may need special care, more investigations and possibly medication to ensure that she can carry the baby safely through to delivery. While pregnancy is a natural condition, it can be complicated even in healthy woman's body because of changes in blood volume, hormone balance, pressure, the physical burden of pregnancy etc. Underlying medical conditions can add even more stress while complications caused by pregnancy itself (such as preeclampsia or gestational diabetes) can turn a normal pregnancy into a high risk pregnancy. A pre-pregnancy visit with a health care provider is especially important for a woman who has a medical problem.

\section{High risk pregnancy assessment: A need for today}

Women form the centre of the family and their health is of prime importance to the wellbeing of the whole family. Women's health is of cardinal importance to the health of the society. In the last decade, considerable attention has been paid to the health of women in their reproductive age by the health care providers and public health experts. The slogans like "Pregnancy is Special, Let's keep it safe" have widely been perpetuated throughout the world. The United Nations Population Fund (UNFPA) estimated that $2,89,000$ women died of pregnancy or child birth related causes in 2013.High risk pregnancy causes almost $20 \%$ of the total burden of disease for women in developing countries. In India pregnancy related deaths of women have declined over the years. The number of maternal deaths per year has come down from approximately 1,00,000 deaths (1991-01) to 44,000 deaths in 2011-13.

Though more than $50 \%$ of reduction has registered in the approximate number of maternal deaths in the last two decades, the present status shows that even now, 120, women die of causes associated with pregnancy, in a day, in India. The life time risk, defined as the probability that one woman of reproductive age (1549) will die due to child birth or puerperium (6 weeks after delivery) assuming that chance of death is uniformly distributed across the entire reproductive span is $0.4 \%$ in India. MDG 5 stipulates that the
MMR level be reduced by3/4 ${ }^{\text {th }}$ between 1990 and 2015. In 1990 the estimated MMR was 437 per 1,00,000 live births. As per the latest Office of Registrar General of India (ORGI) estimates the MMR status is still 167 in 2011-13, it's a slow moving indicator.

Women die from a wide range of complications in pregnancy, child birth or the postpartum period. Many of these complications develop because of their pregnant status and some because pregnancy aggravated an existing disease. The four major killers are: Hemorrhage (27.1\%), Hypertension (14\%), Sepsis (10.7\%), and Obstructed labor. Complications after unsafe abortion cause $13 \%$ of all maternal deaths. Globally about $80 \%$ of maternal deaths are due to these causes. Among the indirect causes (20\%) of maternal death are diseases that complicate pregnancy or are aggravated by pregnancy, such as anemia, HIV. Maternal and New born health are closely linked. It was estimated that approximately 2.7 million new born babies died in 2015 and an addition 2.6 million are still born. A study revealed that India and other developing countries, has a very high perinatal mortality, with a high illiteracy, teeming population and lack of facilities and resources. $70-80 \%$ of perinatal mortality in developing countries including India is accounted for by the mothers falling in the high risk category. This needs for early identification of high risk mothers so that they receive timely and appropriate care. 


\section{Identification of High Risk Pregnancy}

Certain events occurring during the prenatal and intrapartum period can adversely influence the outcome of the infant during postnatal life. This emphasizes the importance of developing technique for identifying the high risk pregnancy. If during prenatal period expectant mothers are screened for their risk factors and grouped and followed up with extra care for those at risk, there will be an impact on the outcome of pregnancy as the high risk pregnancies need specialized investigations and intensive management for the better outcome of mother and the baby. Although identifying risk factors in expectant mothers and special care for them gives health care givers time to anticipate and tackle any adverse situation in time resulting in better neonatal outcome. Yet the adverse neonatal outcome, status of newborn as assessed by APGAR score may be unfavorable even in case of mothers not having any risk factor. It does make sense to use a simple risk assessment scoring system to identify at risk mothers which will help the health care givers to pull in existing means and resources to care more for those in need especially where facilities hardly exist. There were various scoring system tried by different authors trying to relate risk factors present in mothers and their link with the neonatal outcomes.

In 1965, Nesbitt developed Maternal and Child Health Care (MCHC) index based on scoring system whereby disadvantageous clinical features grouped in 10 major categories were given penalty points. In 1969, Nesbitt regrouped abnormal conditions into eight categories. The degree of risk was expressed as a numerical value resulting from the sum of all such penalties subtracted from a perfect score of 100.the patient scoring 70 or less was identified as at risk. The parameters taken were maternal age, parity, past obstetric history, obstetric disorders and nutrition, emotional and socioeconomic survey. In 1969, Aubry, R and Nesbitt R. et al devised a scoring system to objectively evaluate these and other factors such as socioeconomic status, psychological adjustment, age and marital status.Nesbitt and Aubry have developed a semi objective scoring system that assigns a relative score of $0,5,10,15,20,30$ to a number of risk factors. The total score is the result of subtracting the weighted risk of each identified factor from a perfect score of 100. A score of less than 70 indicates considerable risk. Risk factors with a value of 30 or more points are listed below:
a) Abortions (three or more)
b) Fetal death (two or more)
c) Neonatal death (two or more)
d) Syphilis at term
e) Diabetes (all)
f) Hypertension (severe chronic)
g) Hypertension (nephritis)
h) Heart disease (class III, IV)
i) Adrenal, pituitary, thyroid disorder

j) Rh sensitization

k) Severe obesity

l) Prior cesarean section

m) Submucous fibroid

n) Contracted pelvic plane

In 1973, Hobel, CJ et al investigated a high- risk pregnancy screening system based on prenatal and intrapartum factors. Factors were assigned weighed values according to their assumed risks. He included antepartum factors, intrapartum factors, neonatal factors. Total score of prenatal, intrapartum and neonatal period were dichotomized to simply scoring system and less than 10 score was placed in low risk and more than 10 in high risk categories respectively. The relationship between perinatal risk and neonatal risk status was calculated, increasing perinatal risk scores were positively correlated with higher neonatal risk scores.

Pregnancy Risk Assessment: (Tables 1-5).

Table 1.

\begin{tabular}{|c|c|}
\hline OB History Risk Factor & Points \\
\hline Previous stillbirth & 10 \\
\hline Previous neonatal death & 10 \\
\hline Previous premature infant & 10 \\
\hline Post-term > 42 weeks & 10 \\
\hline Fetal blood transfusion for hemolytic disease & 10 \\
\hline Repeated miscarriages & 5 \\
\hline Previous infant > 10 pounds & 5 \\
\hline Six or more completed pregnancies & 5 \\
\hline History of eclampsia & 5 \\
\hline Previous cesarean section & 5 \\
\hline History of preeclampsia & 1 \\
\hline History of fetus with anomalies & 1 \\
\hline
\end{tabular}

Table 2.

\begin{tabular}{|c|c|}
\hline Medical History Risk Factor & Points \\
\hline Abnormal PAP test & 10 \\
\hline Chronic hypertension & 10 \\
\hline Heart disease NYHA Class II-IV (symptomatic) & 10 \\
\hline Insulin dependent diabetes (> A2) & 10 \\
\hline Moderate to severe renal disease & 10 \\
\hline Previous endocrine ablation & 10 \\
\hline Sickle cell disease & 5 \\
\hline Epilepsy & 5 \\
\hline Heart disease NYHA Class I (no symptoms) & 5 \\
\hline History of TB or PPD >=10 mm & 5 \\
\hline Positive serology (for syphilis) & 5 \\
\hline Pulmonary disease & 5 \\
\hline Thyroid disease & \\
\hline
\end{tabular}


Table 3.

\begin{tabular}{|c|c|}
\hline Family History & Points \\
\hline Family history of diabetes & 1 \\
\hline
\end{tabular}

Table 4.

\begin{tabular}{|c|c|}
\hline Physical Risk Factor Risk Factor & Points \\
\hline Incompetent cervix & 10 \\
\hline Uterine malformations & 10 \\
\hline Maternal age 35 and over or 15 and under & 5 \\
\hline Maternal weight $<100$ pounds or $>200$ pounds & 5 \\
\hline Small pelvis & 5 \\
\hline
\end{tabular}

Table 5.

\begin{tabular}{|c|c|}
\hline Current Pregnancy Risk Factor & Points \\
\hline Abnormal fetal position & 10 \\
\hline Moderate to severe preeclampsia & 10 \\
\hline Multiple pregnancy & 10 \\
\hline Placenta abruption & 10 \\
\hline Placenta previa & 10 \\
\hline Polyhydramnios or oligohydramnios & 10 \\
\hline
\end{tabular}

\begin{tabular}{|c|l|}
\hline Excessive use of drugs/alcohol & 5 \\
\hline Gestational diabetes (A1) & 5 \\
\hline Kidney infection & 5 \\
\hline Mild preeclampsia & 5 \\
\hline Rh sensitization only & 5 \\
\hline Severe anemia $<$ 9 g/dL hemoglobin & 5 \\
\hline Severe flu syndrome or viral disease & 5 \\
\hline Vaginal spotting & 5 \\
\hline Bladder infection & 1 \\
\hline Emotional problems & 1 \\
\hline Mild anemia 9 g/dL hemoglobin & 1 \\
\hline Moderate alcohol use & 1 \\
\hline Smoking $>=1$ pack per day & 1 \\
\hline
\end{tabular}

In 1977, Coopland A et al described evaluation of a simple antenatal high risk assessment form. Its ability to assist in high risk selection was measured by applying it retrospectively to antenatal factor of patients. The total risk scores were analyzed in respect to perinatal outcome. As the risk factor increased, the percentage of favorable Apgar ratings decreased. Perinatal mortality increased as risk score increased as did the percentage of neonates requiring special care (Figure 1).

\begin{tabular}{|c|c|c|}
\hline \multicolumn{3}{|c|}{ 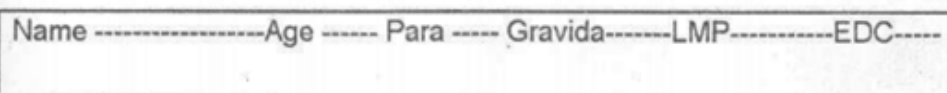 } \\
\hline Reproductive history & $\begin{array}{l}\text { Medical / surgical } \\
\text { conditions }\end{array}$ & Present pregnancy \\
\hline 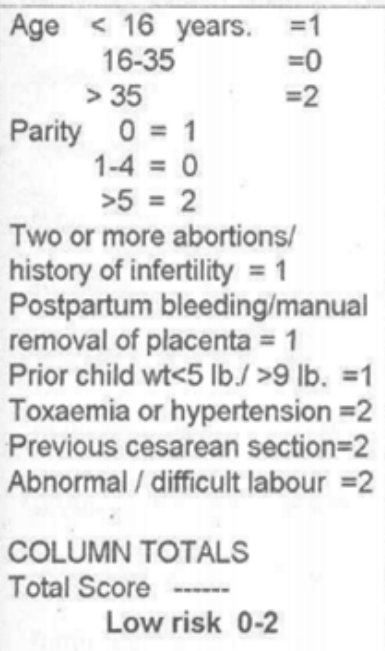 & $\begin{array}{l}\text { Previous gynaec. } \\
\text { surgery = } \\
\text { Chronic renal } \\
\text { decease } \\
\text { Gestational } \\
\text { diabetes }(A)=1 \\
\text { Class } B / \text { greater } \\
\text { diabetes = } \\
\text { Heart disease = } \\
\text { Other significant } \\
\text { medical disorders } \\
\text { (score } 1 \text { to 3 } \\
\text { according to } \\
\text { severity) }\end{array}$ & 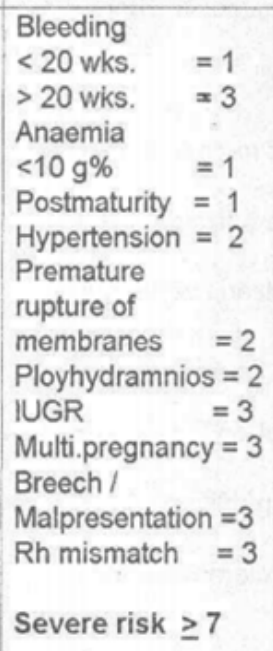 \\
\hline
\end{tabular}

Figure 1: Coopland's High risk evaluation form.

In 1979, Edward evaluated the effectiveness of a simple antepartum risk scoring system, it incorporated demographic, obstetrics, miscellaneous and medical factors, score ranging from 1-10 points for different risk factor. Risk scores obtained for each patient at the first prenatal were updated at 38 weeks of gestation and finally on admission to hospital for labor and delivery. The final risk scores, fetal and neonatal mortality and morbidity were recorded, the data were analyzed to determine the sensitivity, and specificity of the scoring system. Dutta \& Das in 1990 devised a prenatal scoring system which itself was a modification of the high risk scoring system as proposed by Coopland in 1977. According to Dutta \& Das scoring system patients were classified into three groups: Low risk (1-2), Moderate Risk (3-5) and High risk (6 or above). 


\begin{tabular}{|c|c|c|c|c|c|}
\hline Medical risk factors & Score & Past obstetrical factors & Score & Present obstetrical risk factors & Score \\
\hline Age less than 16 & 2 & $\begin{array}{l}\text { Assisted reproductive } \\
\text { technique (ART) conception }\end{array}$ & 1 & \begin{tabular}{|l|} 
Premature rupture of membranes (rupture \\
of membranes before 37 completed weeks)
\end{tabular} & 4 \\
\hline Age more than 35 & 2 & Abortions (first trimester) & 1 & $\begin{array}{l}\text { Preterm labour pains (contractions of four } \\
\text { in } 20 \text { minutes or eight in } 60 \text { minutes plus } \\
\text { progressive change in the cervix/ cervical } \\
\text { dilatation greater than } 1 \mathrm{~cm} / \text { cervical } \\
\text { effacement of } 80 \text { percent of greater) }\end{array}$ & 4 \\
\hline Parity more than 4 & 3 & Abortions (second trimester) & 2 & $\begin{array}{l}\text { Polyhydramnios (amniotic fluid } \\
\text { index on ultrasound is }>24 \mathrm{~cm} \text { ) }\end{array}$ & 4 \\
\hline $\begin{array}{l}\text { Less maternal weight (body mass } \\
\text { index }<19 \text { ) }\end{array}$ & 3 & $\begin{array}{l}\text { Preterm birth (<37 } \\
\text { weeks gestation) }\end{array}$ & 2 & $\begin{array}{l}\text { Intrauterine growth restriction (foetal } \\
\text { growth } 10 \text { th centile for gestational age) }\end{array}$ & 4 \\
\hline $\begin{array}{l}\text { More maternal weight (body } \\
\text { mass index }>28 \text { ) }\end{array}$ & 3 & $\begin{array}{l}\text { Family history of } \\
\text { recurrent abortions }\end{array}$ & 1 & Rhesus isoimmunisation & 4 \\
\hline $\begin{array}{l}\text { Heart disease (symptomatic) New } \\
\text { york heart association class III-IV }\end{array}$ & 4 & $\begin{array}{l}\text { Recurrent spontaneous } \\
\text { abortions }\end{array}$ & 3 & Malpresentation at term & 4 \\
\hline $\begin{array}{l}\text { Heart disease New york heart } \\
\text { association class I-II }\end{array}$ & 1 & Postpartum haemorrhage & 3 & Vaginal bleeding (First trimester) & 2 \\
\hline $\begin{array}{l}\text { Moderate - severe renal disease } \\
\text { (S. creatinine }>1.5 \mathrm{mg} / \mathrm{dl})\end{array}$ & 4 & $\begin{array}{l}\text { Hypertension/ } \\
\text { pre eclampsia }\end{array}$ & 3 & Vaginal bleeding (Second trimester) & 3 \\
\hline $\begin{array}{l}\text { Chronic renal disease } \\
\text { (S.creatinine }>3 \mathrm{mg} / \mathrm{dl} \text { ) }\end{array}$ & 4 & Prolonged labour/difficult delivery & 4 & Mild anemia (Haemoglobin $<10 \mathrm{gm} \%)$ & 1 \\
\hline $\begin{array}{l}\text { Pregestational diabetes mellitus (insulin } \\
\text { dependent or noninsulin dependent) }\end{array}$ & 4 & $\begin{array}{l}\text { Still birth/neonatal } \\
\text { death }\end{array}$ & 4 & $\begin{array}{l}\text { Severe anemia (Haemoglobin } \\
<6 \mathrm{gm} \% \text { ) }\end{array}$ & 4 \\
\hline $\begin{array}{l}\text { Chronic Hypertension (Blood pressure } \\
>140 / 90 \mathrm{~mm} \mathrm{Hg} \text { before pregnancy or } \\
\text { diagnosed before } 20 \text { weeks) }\end{array}$ & 3 & Caesarean delivery & 1 & $\begin{array}{l}\text { Intrahepatic cholestasis of } \\
\text { pregnancy }\end{array}$ & 4 \\
\hline $\begin{array}{l}\text { Controlled epilepsy (at least } 1 \text { year } \\
\text { free of seizures before pregnancy) }\end{array}$ & 1 & $\begin{array}{l}\text { Foetal anomaly with } \\
\text { heritable genetic cause }\end{array}$ & 4 & Minor foetal malformation & 1 \\
\hline $\begin{array}{l}\text { Uncontrolled epilepsy (recent seizure } \\
\text { episode, on multiple antiepileptic) }\end{array}$ & 4 & $\begin{array}{l}\text { Foetal anomaly without } \\
\text { heritable genetic cause }\end{array}$ & 1 & Major foetal malformation & 4 \\
\hline $\begin{array}{l}\text { Active immunological disease like systemic } \\
\text { lupus erythematosus, rheumatoid arthritis, } \\
\text { systemic sclerosis, vasculitis etc }\end{array}$ & 4 & $\begin{array}{l}\text { Radioiodine ablation } \\
\text { within past } 6 \text { months }\end{array}$ & 1 & Placenta praevia & 2 \\
\hline $\begin{array}{l}\text { Immunological disease (inactive } \\
\text { for past } 6 \text { months) }\end{array}$ & 2 & \begin{tabular}{|l|} 
Gynaecological disease like \\
fibroids, synaechiae, ovarian cysts
\end{tabular} & 3 & Morbid adherent placenta & 4 \\
\hline $\begin{array}{l}\text { Tuberculosis or PPD (purified } \\
\text { protein derivative) }>10 \mathrm{~mm}\end{array}$ & 2 & Uterine malformation & 3 & $\begin{array}{l}\text { Gestation hypertension (blood pressure > } \\
\text { 140/90 without proteinuria) }\end{array}$ & 2 \\
\hline $\begin{array}{l}\text { Pulmonary disease like asthma, } \\
\text { pneumonia, bronchitis, pulmonary } \\
\text { tuberculosis }\end{array}$ & 2 & & & $\begin{array}{l}\text { Pre-eclampsia (blood pressure }>140 / 90 \mathrm{~mm} \\
\mathrm{Hg} \text { with proteinuria of }>300 \mathrm{mg} / \mathrm{dl} \text { after } \\
20 \text { weeks of gestation) }\end{array}$ & 3 \\
\hline Smoking & 2 & & & $\begin{array}{l}\text { Eclampsia (Seizures that cannot be } \\
\text { attributed to other causes in a woman } \\
\text { with preeclampsia) }\end{array}$ & 4 \\
\hline Moderate alcohol use & 2 & & & Oligohydramnios & 2 \\
\hline Brain tumour/carcinoma & 4 & & & Abnormal umbilical artery Doppler & 3 \\
\hline Icterus decreasing & 1 & & & Absent or reverse diastolic flows & 4 \\
\hline Icterus increasing & 4 & & & $\begin{array}{l}\text { Gestational diabetes (abnormal } 75 \\
\text { glucose test, } 95 / 180 / 155 \text { ) }\end{array}$ & 3 \\
\hline Chronic disease like HIV & 4 & & & & \\
\hline Positive serology for infections & 2 & & & & \\
\hline $\begin{array}{l}\text { Active bacterial, viral, fungal or } \\
\text { protozoal infection }\end{array}$ & 4 & & & & \\
\hline $\begin{array}{l}\text { Urinary tract infections (urinary culture } \\
\text { with }>10^{\circ} \text { organisms } / \mathrm{mll} \text { ) }\end{array}$ & 2 & & & & \\
\hline
\end{tabular}

Figure 2: Modified high risk pregnancy scoring rate.

Prenatal Scoring Schedule (Dutta and Das): (Table 6)

In 2017 Bhavna Anand et al proposed a new scoring system which is a modification of Coopland et al (1977) and elaborated it further to include various other factors which may have an implication on a woman's obstetrical outcome. Those who fulfilled the required criteria were grouped in three categories; low risk (Group A ) with numerical risk score 0-3, high risk (Group B) with score 4-6 and extremely high risk (Group C) with score $\geq 7$.Patients were then followed till delivery and various maternal and neonatal outcomes were compared between all groups (Figure 2). 
Table 6.

\begin{tabular}{|c|c|c|c|c|c|c|c|}
\hline $\begin{array}{l}\text { Reproductive } \\
\text { History Factors }\end{array}$ & Score & $\begin{array}{c}\text { Past } \\
\text { Obstetrical } \\
\text { History } \\
\end{array}$ & Score & $\begin{array}{l}\text { Present } \\
\text { Pregnancy } \\
\text { Factors }\end{array}$ & Score & $\begin{array}{c}\text { Associated Disease } \\
\text { Factors }\end{array}$ & Score \\
\hline Age & Abortion & 1 & Bleeding $<20$ weeks & 1 & Diabetes & 3 & \\
\hline$<16$ & 1 & $\begin{array}{c}\text { Postpartum } \\
\text { haemorrhage/ } \\
\text { Manual removal } \\
\text { of Placenta }\end{array}$ & 1 & $\begin{array}{l}\text { Bleeding > } 20 \\
\text { weeks }\end{array}$ & 3 & Cardiac disease & 2 \\
\hline $16<35$ & 0 & & & $\begin{array}{c}\text { Anaemia }(\mathrm{Hb}< \\
10 \mathrm{gms})\end{array}$ & 1 & $\begin{array}{c}\text { Previous } \\
\text { gynaecological } \\
\text { surgery }\end{array}$ & 1 \\
\hline$>35$ & 2 & Baby wt $>4 \mathrm{Kg}$ & 1 & Hypertension & 2 & $\begin{array}{l}\text { Chronic renal } \\
\text { disease }\end{array}$ & 2 \\
\hline Parity & Baby wt $<2.5 \mathrm{Kg}$ & 1 & Oedema & 3 & $\begin{array}{l}\text { Infective } \\
\text { hepatitis }\end{array}$ & 1 & \\
\hline 0 & 2 & $\begin{array}{l}\text { Pregnancy } \\
\text { induced } \\
\text { hypertension }\end{array}$ & 1 & Albuminuria & 3 & $\begin{array}{l}\text { Pulmonary } \\
\text { tuberculosis }\end{array}$ & 2 \\
\hline $1-4$ & 0 & Infertility & 1 & $\begin{array}{l}\text { Multiple } \\
\text { Pregnancy }\end{array}$ & 3 & $\begin{array}{l}\text { Other diseases } \\
\text { according to } \\
\text { severity }\end{array}$ & $1-3$ \\
\hline \multirow[t]{6}{*}{5 and above } & 2 & $\begin{array}{l}\text { Previous } \\
\text { Caesarian } \\
\text { section }\end{array}$ & 2 & Breech & 3 & Under -nutrition & 2 \\
\hline & $\begin{array}{l}\text { Still birth/ } \\
\text { Neonatal death }\end{array}$ & 3 & Rh Isoimmunisation & 3 & & & \\
\hline & $\begin{array}{l}\text { Prolonged/ } \\
\text { Difficult labour }\end{array}$ & 1 & Prolonged labour & 1 & & & \\
\hline & & $\begin{array}{l}\text { Premature } \\
\text { rupture of } \\
\text { membranes }\end{array}$ & 2 & & & & \\
\hline & & Polyhydramnios & 2 & & & & \\
\hline & & Small foetus & 1 & & & & \\
\hline
\end{tabular}

\section{High Risk Pregnancy Scoring : A Good Educational Tool}

Haws et al (2009) has reviewed the studies done on impact of pregnancy high risk screening on still births and perinatal mortality and described 10 similar studies done worldwide under different settings with good sensitivity. Perinatal outcomes like preterm births, perinatal mortality, birth asphyxia and low APGAR scores were studied and most of the studies had good sensitivity and correlation of perinatal outcomes in high and extremely high risk patients (Figure 3).

Coopland et al too found that pregnancy with low risk scores were associated with perinatal mortality rates (per 1000 live births) of 4,8 and 6.With an increase in score to between 3 and 6 the perinatal mortality increased to 41.0 ; and with a risk score of 7 or more the rate was 112.0. Samiya et al also found increased risk of low birth weight babies, risk of prematurity and low APGAR scores by using a risk scoring system devised by Dutta \& Das. None of these studies have compared maternal outcomes between the groups, the study done by Bhawna Anand et al have found significant correlation between obstetric hemorrhage requiring blood transfusion and hospital stay in extremely high risk groups as compared to low risk groups (Figure 4).

\section{Conclusion}

High risk pregnancy evaluation provides an opportunity to an obstetrician to identify high risk conditions at the earliest and provide optimal management for optimal maternal and perinatal outcomes. It also provides a close insight of the effect of high risk pregnancy on the perinatal outcomes. Pregnant women require careful follow up for presence of associated maternal and obstetrical high risk factors like gestational diabetes mellitus, preterm delivery and neonatal complication like low APGAR score and prolonged NICU admission; to have optimal outcome. With the rapid advancement of natural history of many new diseases and technology, high risk pregnancy scoring system is the need of today. Timely appropriate care for those who needs most had definite impact on maternal and neonatal outcomes. 


\begin{tabular}{|c|c|c|c|}
\hline Source & Location and Type of Study & Intervention & Stillbirths/Perinatal Outcomes \\
\hline \multicolumn{4}{|l|}{ Observational studies } \\
\hline Abraham et al. 1991 [18] & $\begin{array}{l}\text { India. Health centre setting. } \\
\text { Prospective cohort study. Health } \\
\text { workers at } 6 \text { primary health centres } \\
\text { used a home-based mothers card } \\
\text { with pregnant, mostly illiterate } \\
\text { women }(\mathrm{N}=2446) \text {. }\end{array}$ & $\begin{array}{l}\text { Assessed the association of perinatal } \\
\text { mortality with risk factors recorded on } \\
\text { a home-based mother's card to } \\
\text { pregnant women on which risk factors } \\
\text { and ANC attendance were } \\
\text { documented. }\end{array}$ & $\begin{array}{l}\text { PMR directly related to \# of risk } \\
\text { factors: } \\
0 \text { risk factors: PMR }=25.9 / 1000 \\
\text { I risk factor: PMR }=39.7 / 1000 \\
2 \text { risk factors: PMR }=56.5 / 1000 \\
3 \text { risk factors: PMR } 122.5 / 1000 \text { ) }\end{array}$ \\
\hline Chard et al. $1992[10]$ & $\begin{array}{l}\text { UK. } \\
N=994 \text { pregnant women ( } 470 \\
\text { primiparae; } 524 \text { multiparae) }\end{array}$ & $\begin{array}{l}\text { Used receiver-operating characteristic } \\
\text { curves (ROC) to compare the use of } \\
\text { weighted and unweighted risk scores in } \\
\text { estimating an overall risk score based } \\
\text { on individual risk factors, and relating } \\
\text { this score to fetal outcome. }\end{array}$ & $\begin{array}{l}\text { Weighted risk factor method clearly } \\
\text { superior to unweighted risk factor } \\
\text { method in primiparae. } \\
\text { No difference in multiparae. }\end{array}$ \\
\hline Cho et al. 1991 [163] & $\begin{array}{l}\text { Korea. Chung Ang Medical Center. } \\
\text { Cross-sectional study to test scoring } \\
\text { system. } N=1300 \text { pregnant women } \\
(N=1313 \text { infants) admitted from } \\
1988-1990 \text {. }\end{array}$ & $\begin{array}{l}\text { Assessed the utility of Edwards' scoring } \\
\text { system adapted to a Korean setting in } \\
\text { identifying high-risk pregnancy. Risk } \\
\text { scoring included demographic, } \\
\text { obstetric, medical, and miscellaneous } \\
\text { factors. }\end{array}$ & $\begin{array}{l}560 \text { infants }(42.7 \%) \text { were born to } \\
\text { mothers with risk-scores greater } \\
\text { than } 7 \text {, and } 753 \text { infants }(57.3 \%) \text { were } \\
\text { born to mothers with risk-scores less } \\
\text { than } 7 \text {. }\end{array}$ \\
\hline Lefevre et al. 1989 [15] & $\begin{array}{l}\text { USA. Rural primary care setting. } \\
\text { Prospective study. } N=635 \text { women. } \\
N=47(8.3 \%) \text { adverse outcomes. }\end{array}$ & $\begin{array}{l}\text { Tested the predictive value of } \\
\text { Coopland's obstetric risk in anticipating } \\
\text { adverse outcome (perinatal death, } \\
\text { birthweight }<2500 \text { \&, } 5 \text {-min Apgar } \\
\text { score }<7 \text {, or newborn transferred to a } \\
\text { level } 2 \text { or level } 3 \text { nursery. }\end{array}$ & $\begin{array}{l}\text { There was a clear relationship } \\
\text { between risk score and probability of } \\
\text { adverse outcome. Good sensitivity } \\
\text { could be achieved only at the } \\
\text { expense of a very high false-positive } \\
\text { rate, however. } \\
\text { Risk scoring no more effective than a } \\
\text { policy that would refer all women } \\
\text { with standard obstetric risk factors; } \\
\text { majority of adverse outcomes } \\
\text { occurred in women identified as low. } \\
\text { risk. }\end{array}$ \\
\hline Majoko et al. 2002 [12] & $\begin{array}{l}\text { Zimbabwe. Rural setting. } \\
\text { Evaluation of screening test; sub- } \\
\text { study of ANC trial. } \mathrm{N}=5223 \\
\text { women who received traditional } \\
\text { care from nurse-midwives in } 12 \text { rural } \\
\text { health centres ( } \mathrm{N}=2890 \text { high risk). }\end{array}$ & $\begin{array}{l}\text { Used traditional risk scoring at ANC } \\
\text { booking to group women into low- and } \\
\text { high-risk groups. Highh-risk women were } \\
\text { encouraged to deliver in facilities. }\end{array}$ & $\begin{array}{l}\text { Complications: } 924 \text { (17.7\%) of } \\
\text { women; } 62.4 \% \text { had had risk markers } \\
\text { identified at booking, } 20 \% \text { (577/2890) } \\
\text { of women classified as high risk } \\
\text { developed complications. } \\
\text { Predictive ability of risk allocation: } \\
\text { Likelihood ratio = } 1.16 \text {. }\end{array}$ \\
\hline Mikulandra 1986 [164] & $\begin{array}{l}\text { Croatia. } \\
\text { Prospective study. }\end{array}$ & $\begin{array}{l}\text { Assessed the associations of a risk } \\
\text { factor scale (low, moderate, and high } \\
\text { risk) for pregnancy and delivery on } \\
\text { perinatal outcomes. } \\
\text { High pregnancy risk: } 10.9 \% \text { of cases. } \\
\text { High intrapartum risk: } 14.02 \% \text { of cases. }\end{array}$ & $\begin{array}{l}\text { Severe asphyxia (Apgar } \leq 3) \text { ) } 0.37 \% \text {, } \\
0.81 \% \text {, and } 4.36 \% \text { in low, moderate, } \\
\text { and high-risk groups, respectively (P } \\
<0.001 \text { ). } \\
\text { SBR: } 0.76 \% \text { vs. } 34.48 \% \text { in low vs. high- } \\
\text { risk groups ( } P<0.01 \text { ) }\end{array}$ \\
\hline Morrison 1980 [165] & $\begin{array}{l}\text { USA. } \\
\text { Retrospective analysis. } N=1994 \\
\text { consecutive parturients, } N=472 \\
(23 \% \text { ) high-risk (risk score } \geq 3 \text { ). }\end{array}$ & $\begin{array}{l}\text { Assessed the association of high-risk } \\
\text { (risk score } \geq 3 \text { ) pregnancy with adverse } \\
\text { perinatal outcomes. }\end{array}$ & $\begin{array}{l}\text { PMR: Significantly higher in high-risk } \\
\text { group }(P>0.001) \text {. } \\
\text { Abnormal intrapartum outcome: } 71 \% \\
\text { of high-risk group }(P<0.0001) \text {. }\end{array}$ \\
\hline Morrison 1979 [II] & $\begin{array}{l}\text { USA. } \\
\mathrm{N}=16,733 \text { deliveries. Women } \\
\text { scored during pregnancy using a } \\
\text { simplified, numerical form for } \\
\text { antepartum risk scoring. }\end{array}$ & $\begin{array}{l}\text { Tested the predictive value of a } \\
\text { simplified risk scoring system in } \\
\text { anticipating the risk of perinatal } \\
\text { mortality. }\end{array}$ & $\begin{array}{l}19 \% \text { of group was high-risk (score } \geq \\
\text { 3). } \\
\text { PMR: } 69 / 1000 \text { vs. } 7 / 1000 \text { in high- vs. } \\
\text { low-risk groups, respectively (P< } \\
0.0001 \text { ). } \\
70 \% \text { of perinatal deaths occurred in } \\
\text { high-risk group. }\end{array}$ \\
\hline
\end{tabular}

Figure 3: Impact of pregnancy risk screening on stillbirth and perinatal mortality. 


\begin{tabular}{|c|c|c|c|c|c|c|}
\hline & Total & Frequency & $\%$ & $\begin{array}{c}\text { Odds } \\
\text { Ratio(OR) }\end{array}$ & $\begin{array}{c}95 \% \mathrm{Cl} \\
\text { (confidence } \\
\text { interval) }\end{array}$ & $P$ value \\
\hline \multicolumn{7}{|c|}{ Obstetric haemorrhage requiring blood transfusion } \\
\hline Low Risk & 642 & 4 & $0.62 \%$ & $\begin{array}{c}\text { Reference } \\
\text { (ref) }\end{array}$ & & \\
\hline High Risk & 242 & 11 & $4.55 \%$ & 7.595 & $\begin{array}{l}2.395- \\
24.089\end{array}$ & $<0.001$ \\
\hline $\begin{array}{l}\text { Extremely } \\
\text { High Risk }\end{array}$ & 115 & 20 & $17.39 \%$ & 33.579 & $\begin{array}{l}11.234- \\
100.368\end{array}$ & $<0.001$ \\
\hline \multicolumn{7}{|c|}{ Hospital stay } \\
\hline Low Risk & 642 & 8 & $1.25 \%$ & Ref & & \\
\hline High Risk & 242 & 6 & $2.48 \%$ & 2.015 & $\begin{array}{l}0.692- \\
5.868\end{array}$ & 0.190 \\
\hline $\begin{array}{l}\text { Extremely } \\
\text { High Risk }\end{array}$ & 115 & 17 & $14.78 \%$ & 13.747 & $\begin{array}{l}5.778- \\
32.710 \\
\end{array}$ & $<0.001$ \\
\hline \multicolumn{7}{|c|}{ Need for operative delivery } \\
\hline Low Risk & 642 & 210 & $32.7 \%$ & Ref & & \\
\hline High Risk & 242 & 107 & $44.21 \%$ & 1.352 & $\begin{array}{c}1.0259- \\
1.781\end{array}$ & $<0.001$ \\
\hline $\begin{array}{l}\text { Extremely } \\
\text { High Risk }\end{array}$ & 115 & 82 & $71.30 \%$ & 2.179 & $\begin{array}{l}1.577- \\
3.011\end{array}$ & $<0.001$ \\
\hline
\end{tabular}

Figure 4: Maternal Outcomes.

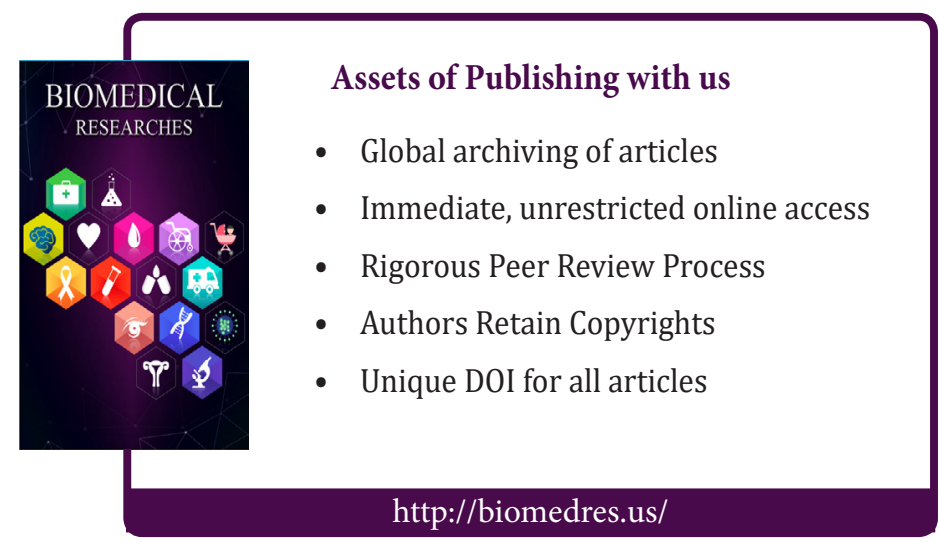

